\title{
ANew Aspect Against Leishmaniasis: Exosomes Obtained from Immune Cells and Parasites
}

\author{
Adil M. Allahverdiyev*, Umut Ogluoglu, Melahat Bagirova, Elif Gençay, Emrah Sefik \\ ABAMOR \\ Yildiz Technical University, Faculty of Chemical and Metallurgical Engineering, Department of Bioengineering, \\ Esenler, Istanbul, Turkey.
}

\begin{abstract}
Despite the struggle against leishmaniasis for many years, the effective treatment method of the disease is not possible and the number of the disease is increasing every year. This is due to the fact that parasites and vectors have developed resistance against medicines and insecticides. Moreover, an efficient vaccine has not been developed yet against leishmaniasis. The importance of exosomes, which are extracellular vesicles secreted by Leishmania parasites, is increasing day by day, as is the case with other infections. Exosomes are being extensively investigated for use in the diagnosis and treatment of some diseases. The number of studies in the literature related to the effect of exosomes on Leishmaniasis is very few. Accordingly, for the first time in this review, important information and suggestions have been given about the importance of exosomes secreted by Leishmania parasites in the struggle against Leishmaniasis and the future treatment plans on this subject. In this paper, we review that how exosomes, which are secreted from Leishmania, effect in the development of the disease, in parasitic virulence, in the triggering of host immune system and in the survival of parasites in the macrophage.
\end{abstract}

Keywords | Leishmania, Exosomes, Vesicles, Macrophage

Editor | Muhammad Imran Rashid, Department of Parasitology, University of Veterinary and Animal Sciences, Lahore, Pakistan.

Received | August 31, 2018; Accepted | October 14, 2018; Published | December 03, 2018

*Correspondence | Adil M. Allahverdiyev, Yildiz Technical University, Faculty of Chemical and Metallurgical Engineering, Department of Bioengineering, Esenler, Istanbul,Turkey; Email: adilmoglu@gmail.com

Citation | Allahverdiyev AM, Ogluoglu U, Bagirova M, Gencay E, Abamor ES (2018). A new aspect against leishmaniasis: exosomes obtained from immune cells and parasites. J. Adv. Parasitol. 5(4): 49-55.

DOI | http://dx.doi.org/10.17582/journal.jap/2018/5.4.49.55

Copyright $(92018$ Allahverdiyev. et al This is an open access article distributed under the Creative Commons Attribution License, which permits unrestricted use, distribution, and reproduction in any medium, provided the original work is properly cited.

\section{INTRODUCTION}

$\mathrm{L}$ eishmania is a parasite of the Kinetoplastida order and the Trypanosomatidae family. Structural and formal changes are also observed in Leishmania, as are other species in this family. Leishmania species have two distinct morphological appearances throughout their life cycle (Claborn, 2010). These forms are the amastigote form found in the host macrophage cells and the promastigot form found in the digestive system of the vector sandfly. The amastigote form is $2 \times 5 \mu \mathrm{m}$ in size, oval and immobile. The promastigote form is a single flageller parasite that can reach up to about $20 \mu \mathrm{m}$ in size and 1.5-3.5 $\mu \mathrm{m}$ in width (McConville et al., 2007). When both forms are stained with giemsa, purple-colored nuclei and a purple rod-shaped kinetoplasty of the parasite are visible (Pérez-Cordero et al., 2011). Leishmaniasis is a serious health problem that spread widely in tropical and subtropical geographical regions, in
98 countries in Europe, Africa, Asia and America (Steverding, 2017). According to the statistics, about 0.2-0.4 million new cases of Visceral leishmaniasis and 0.7-1.2 million new cases of cutaneous leishmania are faced each year (Alvar et al., 2012). Given the close to 300,000 cases visceral form of Leishmaniasis, which can be deadly if not treated, it is clear that the lives of 350 million people living in those areas are at risk (Melo et al., 2017). Depending on the species of leishmania and host factors, infections of leishmaniasis in humans can occur in different forms; these three main forms are cutaneous, mucocutaneous and visceral (Alonso et al., 2016). Cutaneous begin as small papules and within a few weeks they progress to plaque, then painless ulcers and regional lymphadenopathy are observed. In mucocutaneous, bleeding, inflammation, nasal obstruction, mucosal erosion and nasal decay can be observed years after the cutaneous healing. The visceral form targets the tissues and organs of the reticuloendothelial 
system, such as the spleen, liver, bone marrow, and in this form, symptoms such as fever, weight loss, and splenomegaly are observed (Handler et al., 2015). An effective vaccine to prevent the spread of the disease in the treatment of Leishmaniasis has not been developed yet and existing anti-leishmanial drugs are far from treating the disease due to their high cost, toxic effects and the resistance of some parasites to drugs (Abamor et al., 2017).

The parasite is spread by a sandfly to a large mammalian mass including humans and dogs, and the infection develops in macrophages and other cells of the reticuloendothelial system. Throughout the life cycle, leishmania parasites are exposed to many different environments in the infected host (Berg et al., 2013). The cycle begins when the infected host's blood is absorbed by the sandflies. After a small amount of blood, lymph, and macrophages are digested by the sandflies, the parasites that cause Leishmania settle in the middle intestine of adult female sandflies and live there as a compulsory parasite (Mukkada et al., 1985). Leishmania parasites that live extracellularly in the flies alimentary canal migrates into the mammalian host by the bite of the fly, and this is called the host invasion. This process results in the adherence of the parasites to the membrane of macrophages, phagocytosis and subsequent development in phagolysosomes. The promastigote forms of phagocytes parasites cause the entire reticuloendothelial system (spleen, liver) to become infected. The parasites that reach the amastigote form within the macrophage cause the macrophage to burst after a certain period of time due to their cleavage within the phagolysosome. These amastigotes that spread in the environment in this way, when host are bitten by the sandfly again, enter into the sandfly and continue their life cycle (Killick-Kendrick, 1990; Leifso et al., 2007; Walker et al., 2014).

\section{Exosome}

Most eukaryotic cells secrete extracellular vesicles (EV), i.e., apoptotic bodies, microvesicles and exosomes, which can have significant effect on both neighboring and distant cells (Andaloussi et al., 2013; Lee et al., 2012). The largest of the EVs, apoptotic bodies, are formed by apoptotic cell division of a dying cell into closed vesicles and are between $500-4000 \mathrm{~nm}$ in size. Apoptotic bodies are then phagocytosed by macrophages and removed from the medium (Akers et al., 2013). Micro-vesicles (50-1000 nm) are generally smaller than apoptotic bodies and are also known as pouring vesicles or exoveseals. The microvesicles secreted from the surface of the cells express markers indicating changes in the activation state of the apoptosis or origin of the cell. Specifies state of tissue or organ ( $\mathrm{Fi}_{-}$erabracci et al., 2015). Exosomes are small (30-100-nm) vesicles originated from endosomal origin that are released during reticulocyte differentiation as a consequence of the fusion of multivesicular endosomes or multivesicular bod- ies (MVBs) with the plasma membrane (Colombo et al., 2014). These 30-100 nm membrane-bound nanoparticles harboring biomolecular charges present a significant potential in both disease detection and treatment. Exosomes are secreted by many cell types maintained in many organisms, carry signal molecules between cells, and also play an important role in intercellular communication (Keerthikumar et al., 2016). Studies have revealed the therapeutic potential of exosomes, but more work needs to be done to fully demonstrate their efficacy.

Exosomes are produced and secreted by many cell types such as dendritic cells, macrophages, B lymphocytes, T cells, epithelial cells, fibroblasts, adipocytes (Frydrychowicz et al., 2015). In addition, exosomes can be also isolated from body fluids such as saliva, urine, breast milk, semen, blood and bile (Zhang et al., 2016). Vesicles secreted by cells can fit into one of the following states: internalization by other cells, internalization by cells moving away from a significant distance from the oscillating cell, and removal by remote tissues after entering systemic circulation ( $\mathrm{Ra}$ poso et al., 2013). The exosomes secreted from the origin cell contain many proteins such as tetraspanin family proteins (CD9, CD63, CD81, CD82), heat shock proteins (Hsp70, Hsp90), ESCRT complex (Alix, TSG101), fusion proteins (GTPases, flotillin), glycoproteins and lipids such as cholesterol, phosphoglyceride, ceramide. It has also been reported to contain miRNA at high concentration ( $\mathrm{Si}_{-}$ mons et al., 2009; Zheng et al., 2014). The composition of these proteins varies depending on the cell or tissue from the source (Théry, 2011).

\section{Exosomes of Leishmania Parasites}

It was observed that exosomes secreted by various Leishmania species are morphologically and biochemically resemble to mammalian exosomes. However, exosomes secreted by parasites as compared to exosomes of mammalian cells have been reported to account for more than half of the total protein secreted by parasites. It has also been reported that the amount of exosomes released by parasites is 3-4 times higher in the environment with acidic $\mathrm{pH}$ of $37^{\circ} \mathrm{C}$ (Silverman et al., 2010).

Based on the literature review, against Leishmaniasisit has been found thatthere are 3 different roles of exosomes secreted by Leishmania parasites and immune cells. To these; (a) the development of infection and the formation of virulence, (b) the triggering of the host immune system, and (c) the ability to sustain life in host macrophages are included.

\section{a.The Role of Exosomes in the Development of Leish-} mania Infection and in the Formation of Virulence: Macrophages, composed of blood, spleen and bone marrow monocytes, play important role in the homeostasis, repair of the organism, and in particular the immune response 
to pathogens (Wynn et al., 2013). Macrophage activation occurs in two ways, classical and alternative.In classical activation, $\mathrm{CD} 4+\mathrm{Th} 1$ cells produce nitric oxide synthase (iNOS), an enzyme that catalyses $\mathrm{L}$-arginine production to produce nitric oxide by producing IFN- $\gamma$. It has also been found that besides IFN- $\gamma$, cytokines such as IL-1, TNF and IFN- $\alpha$ also lead to macrophage activation and increase the expression of iNOS leading to NO production (Bronte et al., 2005). In alternative activation, macrophage activation is stimulated by CD 8 + Th2 cytokines such as IL-4, IL-10 and IL-13. It has been shown that IL-4-induced polyamine biosynthesis supports the survival of $L$. major parasites in macrophages and IL-10 inhibits inflammatory cytokine production, particularly TNF (Gordon, 2003; Kropf et al., 2005). Also, Leishmania parasites survive by introducing various defense systems in phagocytes or macrophages they are involved in. These are possible thanks to the surface immunogens of the parasites, glycoprotein 63 (GP63) and lipophosphoglycan (LPG). GP63, also called zinc-metalloprotease, is a Leishmania surface protein with a size of $60-66 \mathrm{kDa}$ that protects intracellular amastigotes in host macrophages, acts in the escape mechanisms of the parasite from the macrophages and plays an effective role to infect the host (Isnard et al., 2012). LPG is a glycoconjugate commonly expressed on the surface of Leishmania promastigotes. LPG is involved in a variety of functions such as recognition, resistance to complement, modulation of nitric oxide (NO) and IL-12 production, phagocytosis and protection from vacuoles in the acidic environment (Paranaíba et al., 2015).These molecules promote survival of macrophages in parasites by the inhibition of oxidative respiratory metabolites of hydrolytic enzymes in phagolysosomes and the inhibition of phagosome-endosome fusion by LPG molecules. In addition, the alteration of calcium mobilization on the macrophage by parasites also leads to the inhibition of signal transduction and host signaling pathways. It also by providing the modulation of macrophage cytokine production and the inhibition of $\mathrm{NO}$ production by glycosylinositolphospholipids (GILPs) on the amastigote surface, allowing the parasites to survive in the macrophage phagolysosome (Cunningham, 2002; Descoteaux et al., 1991; Descoteaux et al., 1999; Kane et al., 2000; Miao et al., 1995; Olivier et al., 1992; Reiner, 1994; Roberts et al., 1996; Turco et al., 1992).

One study in literature indicated dissimilar inhibition of macrophane from NO mechanism. According to the result of this study, the host cells of Leishmania donovani ( $L$. donovani) leads to increase Src-homology 2 domain comprising $L$. donovani host cells phosphatase-1 (SHP-1) and other protein tyrosine phosphatase (PTP) activity as potential (Blanchette et al., 1999). SHP-1 which was fastly activated by L.donovani promastigotes causes inhibition of induced JAK2 phosphorylation with IFN- $\gamma$ (Shio et al., 2012). As a result of this situation, it was observed that
SHP-1 breaks the functions of nitric oxide-dependent and independent microbicidal mechanisms and is important for the survival of the mammalian L. donovaniand for spreading the disease. There are two protein activation for this situation. Fructose 1,6-biphosphate aldolase in L.donovani and elongation factor- $1 \alpha$ (EF-1 $\alpha$ ) SHP-1 bonding as activating protein were detected (Nandan et al., 2007; Nandan et al., 2002). However, studies in recent years has reported that exosome also have role for the survival of Leishmania parasites in macrophages. In one study, as a result of investigating of Leishmania exosome, it was mentioned that exosome make easier communication between the host cell and carry these proteins. This situation was supported by the detection of aldolase and EF- $1 \alpha$ in the cytosol of infected cells as well as the exosomal markers of HSP70 and HSP90, heat shock protein, which conferred temperature resistance to parasites. In addition, the fact that leismania exosomes induces secretion of IL-8 was clearly showed and this hypotesis could help enfection of the host cell by Leismania parasites (Silverman et al., 2010).

Molecular interaction between parasite and host cell is imported in infection therefore; success in one infection depends on the ability of parasite to escape the defense mechanisms of the host cell and the invasion and reproduction of the target cell (da Fonseca Pires et al., 2014). After that exosomes carry virulence factors like LPG,G$\mathrm{P} 63, \mathrm{P} 46$ was shown in studies at subsequent years belonging to leismania parasite exosomes, the studies are related to transportation of these virulance factor via exosomes and their effect. There are studies in the literature that are vital for exosomes which is released by pathogenic infections. They are of great interest and importance because they are believed to play a role in vasculitis, especially in host-pathogen interactions, where organisms are secreted. One study according to this information was the role of wild type (WT) and GP63(-/-) knock-out (KO) Leishmania exosomes on modulation of inflammatuar response. It was seen that Leishmania exosomes have shared some immunomodulatory properties with Leishmania parasites. $\mathrm{KO}$ parasites have ability to low immun regulation as compared with WT parasites. The control of this situation was mediated by GP63 cleavage of the macrophage protein tyrosine phosphatases (PTP), and also reduction of translocation of pro-inflammatory transcription factors (TF) and reduction of proinflammatory gene expression. Many receptors and correlator like IL-1 reseptör type I, Nlrc4, Treml and Dectin-1 (Clec7a) were downregulated after only enfection of WT parasites (Hassani et al., 2014). The first time in L. major is a $46-\mathrm{kD}$ protein whose progeny is unknown, its function is unknown, and accumulates in the cytoplasm of infected macrophages. In this situation, it was detected that increasing of wild type L.major lesion production and $\mathrm{P} 46$ overexpression and a non-virulent HSP100 null mutant compensates for the loss of viru- 
lence (Bifeld et al., 2015). In a study conducted, exosomes were isolated from $L$. major to determine whether P46 was a virulence factor. Bone marrow-derived macrophages (BMMs) were first incubated with exosomes derived from L. major WT, L. major P46 - / -, L. major P46 - / - / + and Leishmania-free medium, then were infected with $L$. major. The results of in vitro infection confirm the effective increase of exosomes, parasite load, in the increased parasitic load on the medium-treated BMMs. The $\mathrm{P} 46$ protein has been shown to be an exosomal protein due to the Hsp100 heat shock protein as it has almost the same effect in other experimental groups (Bifeld et al., 2015).

In a study in which Atayde et al. performed with L. infantum and L. major, it was seen that the promastigotes in the midgut of the sandfly secrete exosomes, and these exosomes were given with parasites during the bite of the sandfly. In the same study, mice were harvested at week 5 post-infection with drainage lymph nodes and significantly elevated levels of IL-4 and IFN- $\gamma$ have been observed that support increased parasite replication when their cytokine mRNA levels are measured. It has been determined that mice injected exosomes with $L$. major exacerbate the disease, increase lesion formation significantly, increase leishmanian virulence factors, and regulate the immune system by expressing IL-4 and IFN- $\gamma$ (Atayde et al., 2015).

Different mitochondrial proteins are found in Leishmania secretomy and exosome related to Leishmania parasite. In another study, chaperonin 10 (CPN10) mitochondrial protein in L. donovani was examined for the transport of cytokines via the exosomes of infected macrophages. THP-1 cells, a human monocytic cell line differentiated into macrophages, were infected with $L$. donovani and were seen in the cytosol of macrophages when 4 ', 6-diamidino-2-phenylindole (DAPI) staining was applied. (Genin et al., 2015). Leishmania CPN10 regulated expression of Leishmania proteome at the same time macrophages proteins in infected cells affected the expression of a large and different group. As a result, $\mathrm{CPN} 10$ has been shown to attenuate the rate of leishmania ingestion, both in promoting intracellular survival and limiting the extent of macrophage activation (Colineau et al., 2017).

\section{b. The role of exosomes in triggering the host immune} system against Leishmaniasis: One of the other important problems against Leishmaniasis is that there is no effective vaccine. Although to the development of vaccines against Leishmaniasis several approaches have been developed by now but it is not possible to obtain an effective vaccine yet. At the recent years, studies on exosomes related to Leishmania shows that exosomes have the potential to develop vaccines against Leishmaniasis. Exosomes that derived from Leishmania lead to promising hope in vaccine development. In the research on dendritic cells,
Schnitzer et al. in the study on Balb/c mice they considered that exosomes of bone marrow dendritic cells were loaded with L. major antigen and activated with $\mathrm{CpG}$ containing oligodeoxynucleotides ( $\mathrm{CpG} \mathrm{ODN})$. After one week it has been found that exosomes released from dendritic cells of the L. majorinfected mice help protective immunity against cutaneous leishmaniasis. In the same study it has also been shown that exosomes secreted by dendritic cells which fixed by paraformaldehyde or exposed to UV radiation or broken down can induce $T$ cell proliferation and IL-12 secretion. As a result of the study, it has also been found that this protective effect does not depend on cell morphology or cell viability (Schnitzer et al., 2010). However, another study found that exposure of human monocytes to Leishmania exosomes resulted in an increase in IL-10 production, a change in monocyte-cytokine responses, inhibition of IL-8 and TNF-a production, and this response was anti-inflammatory. Again, in this study, the results of infected monocytes against naive monocytes exposed to exosomes and IFN- $\gamma$ at various doses were compared. The results have shown that exosomes inhibit cytokine production at a high level, but inhibition of this cytokine production requires exosomes at very high concentrations. Moreover, exosomes from WT L. donovani have prevented the differentiation of naive CD4 T cells into IFN- $\gamma$ producing Th1 cells. Exosomes from L. major have also been examined to check that immunosuppressive properties are not specific to $L$. donovani exosomes. As a result, it has been observed that these exosomes increase Th2 cell polarization in vivo and cause leishmania infection to exacerbate (Silverman et al., 2010).

The vaccine development against Leishmaniasis is not limited to dendritic cells alone. An important member of the immune system, and studies of macrophages, which act as a host in Leishmania parasites, have also been studied. In one study, the relationship between macrophages and Leishmania parasites was examined. The J774 mouse macrophage cell line infected with L. mexicana (LEISHX) or LPS-stimulated (LPSX) or non-treated (NILX) was separately incubated with each other. After incubation, proteomic analysis of exosomes released from the J774 mouse macrophage cell line was performed. Analysis showed that approximately $80 \%$ of the Leishmania proteins were shared between the NILX, LPSX and LEISHX exosomes, and these exosomes were generally found to aid in cellcell communication, vesicle exchange and signaling. It has also been shown that the only virulence factor found in exosomes of these macrophages is Leishmania GP63 and can reach the cytoplasm or the nucleus of the macrophage (Hassani et al., 2013).

c. Leishmania exosomes and resistance mechanism: The most common and most appropriate option for the treatment of leishmaniasis is pentavalent antimony based drug 
therapy. One of the problems that arise in the fight against the disease is the development of resistance to these drugs used in parasites. Although there are various explanations for the development of resistance to drugs used in parasites, it is not fully adequate. There are $58 \mathrm{kDa}$ protein Antimony Resistance Marker 58 kDa (ARM58) codes which impart antimony III (SbIII) resistance to promastigotes and antimony $\mathrm{V}(\mathrm{SbV})$ resistance to intracellular amastigotes in species such as L. donovani and L. braziliensis (Schäfer et al., 2014). Recent studies show that exosomes play a role in the resistance of Leishmania parasites. In one study, $L$. donovani exosomes played a role in resistance to the host defense mechanism, and ARM58 protein was found in these exosomes. In addition, the ARM58 / ARM56 mediated antimony resistance mechanism has been expressed when overexpressed. At the same time, it was determined that the infectivity of parasites has been increased in vitro (Nevado et al., 2016).

In study conducted, The HSP90 chaperone and its cochaperons Aha1 (Bartsch et al., 2017) and P23 (Hombach et al., 2015), a heat-shock protein found in the exosomes of $L$. donovani, are important for the survival, life cycle control, infectious and stress tolerance of these parasites and therapeutic intervention against potential targets. The lack of Aha1 leads to the decreasing of parasites in higher temperature in vitro, hypersensitive of ethanol and the transportation of the plasma membrane to be damaged. The lack of P23 increase ATPase activation of HSP90 and causes sensitive phenotype to Radicicol drug which is HSP90 inhibitor. As a result of this, these cochaperones that are secreted by the exosomes and steered to the host cell cytosol have been shown to have an indirect effect on viability by interfering with the activity of the HSP90 inhibitors (Bartsch et al., 2017; Hombach et al., 2015).

\section{Conclusion and Future Prospects}

Studies through extracellular vesicles are becoming increasingly important in research on Leishmaniasis as in other diseases in recent years. Exosomes of the Leishmania parasite have also been found to contain proteins and nucleic acids of the original cell as a result of these studies. Review of the literature has focused on the role of exosomes secreted by Leishmania parasites in the development of Leishmaniasis, the survival of Leishmania in host macrophage, resistance to the host immune system and its role in triggering the host immune system. However, the studies carried out in this direction are few and the results obtained are inadequate and contradictory. In addition, studies predominantly carried out in this area include L. donovani, L. major species of Leishmania parasites. We did not find much information against other species. Since there is no information on the isolation of exosomes from species such as L. tropica, L. infantum, which are other important species of Leishmania parasites, it is important to continue their studies in the future. Although there is information on the use of exosomes obtained from other infections in diagnosis, there are no studies on this in Leishmaniasis. Exosomes can also be used in the diagnosis of Leishmaniasis. Therefore, diagnostic values of the exosomes to be isolated should be revealed in a short time.

The published literature suggests that exosomes isolated from either Leishmania parasites or from immune system cells, may trigger an immune response and thus play an important role in the immunotherapy of leishmaniasis. However, due to the low number of studies conducted on this area, more extensive studies are needed. On the other hand, studies conducted in recent years are attempting to clarify the role of exosomes in the immunopathogenesis of Leishmania parasites. With the increase of studies in this area, the exosomes will be clear about the survival of parasites, infections and the activity of making the disease. Thus, significant potentials will be identified in studies of exosomes, in the diagnosis of Leishmaniasis, immunotherapy and vaccine studies. It has also been reported that exosomes have an active role in resistance to parasites against anti-leishmanial drugs used. Along with the clarification of this resistance mechanism, new approaches can be developed in the treatment of the disease. However, due to their chemical structure, the isolated exosomes present a great potential to be used as nanoparticulate drug delivery systems in the treatment of leishmaniasis and other infectious diseases. All these expectations can be made possible by the increased interest shown in the relevant work. As a consequence, since exosomes have a great potential to fight against Leishmaniasis, the maintenance of these studies is of strategic importance.

\section{ACKNOWLEDGEMENTS}

There is no colleagues that author at their acknowledgements.

\section{CONFLICT OF INTEREST}

There is no conflict of interest.

\section{AUTHORS CONTRIBUTUION}

Each author contributed to all sections of the article.

\section{REFERENCES}

-Abamor ES, Allahverdiyev AM, Bagirova M, Rafailovich M (2017). Meglumine antımoniate-TiO2@ Ag nanoparticle combinations reduce toxicity of the drug while enhancing its antileishmanial effect. Acta Trop.169:30-42. https://doi. org/10.1016/j.actatropica.2017.01.005

- Akers JC, Gonda D, Kim R, Carter BS, Chen CC (2013). 
Biogenesis of extracellular vesicles (EV): exosomes, microvesicles, retrovirus-like vesicles, and apoptotic bodies. J. Neuro-oncol.113(1):1-11. https://doi.org/10.1007/ s11060-013-1084-8

- Alonso G, Rastrojo A, López-Pérez S, Requena JM, Aguado B (2016). Resequencing and assembly of seven complex loci to improve the Leishmania major (Friedlin strain) reference genome. Parasit. Vectors. 9(1):74. https://doi.org/10.1186/ s13071-016-1329-4

- Alvar J, Velez ID, Bern C (2012). Leishmaniasis worldwide and global estimates of its incidence. PloS one.7(5):e35671 https://doi.org/10.1371/journal.pone.0035671.

- Andaloussi SE, Mäger I, Breakefield XO, Wood MJ (2013). Extracellular vesicles: biology and emerging therapeutic opportunities. Natur. Rev. Drug Discov. 12(5):347. https:// doi.org/10.1038/nrd3978

- Atayde VD, Aslan H, Townsend S. (2015). Exosome secretion by the parasitic protozoan Leishmania within the sand fly midgut. Cell Rep.13(5):957-67. https://doi.org/10.1016/j. celrep.2015.09.058

- Bartsch K, Hombach-Barrigah A, Clos J (2017). Hsp90 inhibitors radicicol and geldanamycin have opposing effects on Leishmania Aha1-dependent proliferation. Cell Stress Chaper. 22(5):729-42. https://doi.org/10.1007/s12192017-0800-2

- Berg M, Mannaert A, Vanaerschot M, Van der Auwera G, Dujardin J-C (2013). (Post-) Genomic approaches to tackle drug resistance in Leishmania. Parasitology. 140(12):1492505. https://doi.org/10.1017/S0031182013000140

- Bifeld E, Chrobak M, Zander D (2015). Geographical sequence variation in the Leishmania major virulence factor P46. Infect. Genet. Evol. 30:195-205. https://doi. org/10.1016/j.meegid.2014.12.029

- Bifeld E, Clos J (2015). The genetics of Leishmania virulence. Med. Microbiol. Immunol. 204(6):619-34. https://doi. org/10.1007/s00430-015-0422-1

- Blanchette J, Racette N, Faure R, Siminovitch KA, Olivier $M$ (1999). Leishmania-induced increases in activation of macrophage SHP-1 tyrosine phosphatase are associated with impaired IFN- $₫$-triggered JAK2 activation. Euro. J. Immunol. 29(11):3737-44. https://doi.org/10.1002/ (SICI)1521-4141(199911)29:11\%3C3737::AIDIMMU3737\%3E3.0.CO;2-S

- Bronte V,Zanovello P (2005). Regulation of immune responses by L-arginine metabolism. Nat. Rev. Immunol. 5(8):641. https://doi.org/10.1038/nri1668

- Claborn DM (2010). The biology and control of leishmaniasis vectors. J. Glob. Infect. Dis. 2(2):127. https://doi. org/10.4103/0974-777X.62866

- Colineau L, Clos J, Moon K-M, Foster LJ, Reiner NE (2017). Leishmania donovani chaperonin 10 regulates parasite internalization and intracellular survival in human macrophages. Med. Microbiol. Immunol. 206(3):235-57. https://doi.org/10.1007/s00430-017-0500-7

- Colombo M, Raposo G, Théry C (2014). Biogenesis, secretion, and intercellular interactions of exosomes and other extracellularvesicles. Ann. Rev. Cell Develop.Biol.30:255-89. https://doi.org/10.1146/annurev-cellbio-101512-122326

- Cunningham AC (2002). Parasitic adaptive mechanisms in infection by Leishmania. Exper. Mole. Pathol. 72(2):132-41 https://doi.org/10.1006/exmp.2002.2418.

- da Fonseca Pires S, Fialho Jr LC, Silva SO (2014).Identification of virulence factors in Leishmania infantum strains by a proteomic approach. J. Prot. Res. 13(4):1860-72. https://doi. org/10.1021/pr400923g

- Descoteaux A, Turco S, Sacks D, Matlashewski G (1991). Leishmania donovani lipophosphoglycan selectively inhibits signal transduction in macrophages. J. Immunol. 146(8):2747-53.

- Descoteaux A,Turco SJ (1999). Glycoconjugates in Leishmania infectivity. Biochimica et Biophys. Acta (BBA)-Mole. Basis Dis.1455(2-3):341-52. https://doi.org/10.1016/S09254439(99)00065-4

- Fierabracci A, Del Fattore A, Luciano R (2015). Recent advances in mesenchymal stem cell immunomodulation: the role of microvesicles. Cell Transplant. 24(2):133-49. https:// doi.org/10.3727/096368913X675728

- Frydrychowicz M, Kolecka-Bednarczyk A, Madejczyk M, Yasar S, Dworacki G (2015). Exosomes-Structure, Biogenesis and Biological Role in Non-Small-Cell Lung Cancer. Scandinavian J. Immunol. 81(1):2-10. https://doi. org/10.1111/sji.12247

- Genin M, Clement F, Fattaccioli A, Raes M, Michiels C (2015). M1 and M2 macrophages derived from THP1 cells differentially modulate the response of cancer cells to etoposide. BMC Cancer. 15(1):577. https://doi. org/10.1186/s12885-015-1546-9

- Gordon S (2003). Alternative activation of macrophages. Nat. Rev. Immunol. 3(1):23. https://doi.org/10.1038/nri978

- Handler MZ, Patel PA, Kapila R, Al-Qubati Y, Schwartz RA (2015). Cutaneous and mucocutaneous leishmaniasis: differential diagnosis, diagnosis, histopathology, and management. J. American Acad. Dermatol. 73(6):911-26. https://doi.org/10.1016/j.jaad.2014.08.051

- Hassani K, Olivier M (2013). Immunomodulatory impact of leishmania-induced macrophage exosomes: a comparative proteomic and functional analysis. PLoS neglected Trop. Dis. 7(5):e2185. https://doi.org/10.1371/journal.pntd.0002185

- Hassani K, Shio MT, Martel C, Faubert D, Olivier M (2014). Absence of metalloprotease GP63 alters the protein content of Leishmania exosomes. PloS one. 9(4):e95007. https://doi. org/10.1371/journal.pone.0095007

- Hombach A, Ommen G, Sattler V, Clos J (2015). Leishmania donovani P23 protects parasites against HSP90 inhibitormediated growth arrest. Cell Stress Chaper. 20(4):673-85. https://doi.org/10.1007/s12192-015-0595-y

- Isnard A, Shio MT, Olivier M (2012). Impact of Leishmania metalloprotease GP63 on macrophage signaling. Frontiers Cellul. Infect. Microbiol. 2:72. https://doi.org/10.3389/ fcimb.2012.00072

- Kane MM, Mosser DM (2000). Leishmania parasites and their ploys to disrupt macrophage activation. Curr. Opin. Hematol. 7(1):26-31. https://doi.org/10.1097/00062752200001000-00006

- Keerthikumar S, Chisanga D, Ariyaratne D (2016). ExoCarta: a web-based compendium of exosomal cargo. J. Mole. Biol. 428(4):688-92. https://doi.org/10.1016/j.jmb.2015.09.019

- Killick-Kendrick R (1990). The life-cycle of Leishmania in the sandfly with special reference to the form infective to the vertebrate host. Ann. Parasitol. Hum. Compar. 65:37-42. https://doi.org/10.1051/parasite/1990651037

- Kropf P, Fuentes JM, Fähnrich E (2005). Arginase and polyamine synthesis are key factors in the regulation of experimental leishmaniasis in vivo. FASEB J. 19(8):1000-2.

- Lee Y, El Andaloussi S, Wood MJ (2012). Exosomes and microvesicles: extracellular vesicles for genetic information 
transfer and gene therapy. Human Mole. Genet. 21(R1):R125-R34. https://doi.org/10.1093/hmg/dds317

- Leifso K, Cohen-Freue G, Dogra N, Murray A, McMaster WR (2007). Genomic and proteomic expression analysis of Leishmania promastigote and amastigote life stages: the Leishmania genome is constitutively expressed. Mole. Biochem. Parasitol. 152(1):35-46. https://doi.org/10.1016/j. molbiopara.2006.11.009

- McConville MJ, De Souza D, Saunders E, Likic VA, Naderer $T$ (2007). Living in a phagolysosome; metabolism of Leishmania amastigotes. Trends Parasit. 23(8):368-75. https://doi.org/10.1016/j.pt.2007.06.009

- Melo GD, Goyard S, Lecoeur H (2017). New insights into experimental visceral leishmaniasis: Real-time in vivo imaging of Leishmania donovani virulence. PLoS Neglect. Trop. Dis.11(9):e0005924. https://doi.org/10.1007/s13244017-0573-2

- Miao L, Stafford A, Nir S (1995). Potent inhibition of viral fusion by the lipophosphoglycan of Leishmania donovani. Biochemistry. 34(14):4676-83. https://doi.org/10.1021/ bi00014a022

- Mukkada AJ, Meade JC, Glaser TA, Bonventre PF (1985). Enhanced metabolism of Leishmania donovani amastigotes at acid $\mathrm{pH}$ : an adaptation for intracellular growth. Science. 229(4718):1099-101. https://doi.org/10.1126/ science. 4035350

- Nandan D, Tran T, Trinh E, Silverman JM, Lopez M (2007). Identification of leishmania fructose-1, 6-bisphosphate aldolase as a novel activator of host macrophage Src homology 2 domain containing protein tyrosine phosphatase SHP-1. Biochem. Biophy. Res. Commun. 364(3):601-7. https://doi. org/10.1016/j.bbrc.2007.10.065

- Nandan D, Yi T, Lopez M, Lai C, Reiner NE (2002). Leishmania EF-1区 activates the Src homology 2 domain containing tyrosine phosphatase SHP-1 leading to macrophage deactivation. J. Biolog. Chem. 277(51):501907. https://doi.org/10.1074/jbc.M209210200

- Nevado PT, Bifeld E, Höhn K, Clos J (2016). A telomeric cluster of antimony resistance genes on chromosome 34 of Leishmania infantum. Antimicrob. Agents Chemother. 60(9):5262-75. https://doi.org/10.1128/AAC.00544-16

- Olivier M, Baimbridge K, Reiner N (1992). Stimulusresponse coupling in monocytes infected with Leishmania. Attenuation of calcium transients is related to defective agonist-induced accumulation of inositol phosphates. J. Immunol. 148(4):1188-96.

- Paranaíba LF, de Assis RR, Nogueira PM (2015). Leishmania enriettii: biochemical characterisation of lipophosphoglycans (LPGs) and glycoinositolphospholipids (GIPLs) and infectivity to Cavia porcellus. Parasit. Vectors. 8(1):31.

- Pérez-Cordero J-J, Sánchez-Suárez J, Delgado G (2011). Use of a fluorescent stain for evaluating in vitro infection with Leishmania panamensis. Exper. Parasitol. 129(1):31-5. https://doi.org/10.1016/j.exppara.2011.05.022

- Raposo G, Stoorvogel W (2013). Extracellular vesicles: exosomes, microvesicles, and friends. J. Cell Biol. 200(4) 373-83. https://doi.org/10.1083/jcb.201211138

- Reiner NE (1994). Altered cell signaling and mononuclear phagocyte deactivation during intracellular infection. Immunol. Today.15(8):374-81. https://doi. org/10.1016/0167-5699(94)90176-7

- Roberts LS, Janovy J, Schmidt GD (1996). Foundations of parasitology: Wm. C. Brown Dubuque.

- Schäfer C, Nevado PT, Zander D, Clos J (2014). Reduced antimony accumulation in ARM58-overexpressing Leishmania infantum. Antimicrob. Agents Chemother. 58(3):1565-74. https://doi.org/10.1128/AAC.01881-13

- Schnitzer JK, Berzel S, Fajardo-Moser M, Remer KA, Moll H (2010). Fragments of antigen-loaded dendritic cells (DC) and DC-derived exosomes induce protective immunity against Leishmania major. Vaccine. 28(36):5785-93. https:// doi.org/10.1016/j.vaccine.2010.06.077

- Shio MT, Hassani K, Isnard A (2012). Host cell signalling and leishmania mechanisms of evasion. J. Trop. Med. 2012.

- Silverman JM, Clos J, de'Oliveira CC (2010). An exosomebased secretion pathway is responsible for protein export from Leishmania and communication with macrophages. J. Cell Sci.123(6): 842-52.https://doi.org/10.1242/jcs.056465

- Silverman JM, Clos J, Horakova E (2010). Leishmania exosomes modulate innate and adaptive immune responses through effects on monocytes and dendritic cells. J. Immunol. 185(9): 5011-22. https://doi.org/10.4049/ jimmunol.1000541

- Simons M, Raposo G (2009). Exosomes-vesicular carriers for intercellular communication. Curr. Opin. Cell Biol. 21(4):575-81. https://doi.org/10.1016/j.ceb.2009.03.007

- Steverding D (2017). The history of leishmaniasis. Parasit. Vectors.10(1):82. https://doi.org/10.1186/s13071-0172028-5

- Théry C (2011). Exosomes: secreted vesicles and intercellular communications. F1000 biology reports. 3. https://doi. org/10.3410/B3-15

- Turco SJ, Descoteaux A (1992). The lipophosphoglycan of Leishmania parasites. Ann. Rev. Microbiol. 46(1):65-92. https://doi.org/10.1146/annurev.mi.46.100192.000433

- Walker DM, Oghumu S, Gupta G (2014). Mechanisms of cellular invasion by intracellular parasites. Cellul. Mole. Life Sci. 71(7):1245-63. https://doi.org/10.1007/s00018-0131491-1

- Wynn TA, Chawla A, Pollard JW (2013). Macrophage biology in development, homeostasis and disease. Nature. 496(7446):445. https://doi.org/10.1038/nature12034

- Zhang H-G, Cao P, Teng Y (2016). Isolation, identification, and characterization of novel nanovesicles. Oncotarget. 7(27):41346. https://doi.org/10.18632/oncotarget.9325

- Zheng X, Chen F, Zhang J, Zhang Q, Lin J (2014). Exosome analysis: a promising biomarker system with special attention to saliva. J. Memb. Biol. 247(11):1129-36. https:// doi.org/10.1007/s00232-014-9717-1 